\title{
Muon excess in ultra-high energy inclined EAS according to the NEVOD-DECOR data
}

\author{
R.P. Kokoulin ${ }^{a}{ }^{*}$, N.S. Barbashina ${ }^{a}$, A.G. Bogdanov $a$, S.S. Khokhlov ${ }^{a}$, \\ V.V. Kindin $a$, K.G. Kompaniets ${ }^{a}$, G. Mannocchi ${ }^{b}$, A.A. Petrukhin $a$, V.V. Shutenko ${ }^{a}$, \\ G. Trinchero $^{b}$, I.I. Yashin $^{a}$, E.A. Yurina $^{a}$ and E.A. Zadeba $a$ \\ a National Research Nuclear University MEPhI (Moscow Engineering Physics Institute), \\ 115409, Moscow, Russia \\ b Osservatorio Astrofisico di Torino - INAF, \\ 10025, Torino, Italy \\ E-mail: rpkokoulin@mephi.ru
}

Data of the NEVOD-DECOR experiment on investigations of inclined cosmic ray muon bundles for a long time period (May 2012 - March 2021) are presented. Their comparison with the results of calculations based on simulations of EAS muon component allows one to study the behavior of the energy spectrum and mass composition of primary cosmic rays and/or to check the validity of hadron interaction models in a wide energy range from about $10^{16}$ to more than $10^{18} \mathrm{eV}$. The analysis showed that the observed intensity of muon bundles at primary particle energies of about $10^{18} \mathrm{eV}$ and higher can be compatible with the expectation only under the assumption of an extremely heavy mass composition of cosmic rays. This conclusion is consistent with data of a number of other experiments investigating the muon component of air showers at ultra-high energies. On the contrary, measurements of the depth of the shower maximum in the atmosphere $\left(X_{\max }\right)$ in the experiments using air fluorescence technique favor a light mass composition of primary cosmic rays at these energies. This contradiction (so-called "muon puzzle") cannot be resolved without serious changes of the existing hadron interaction models.

\section{"Presenter}




\section{Introduction}

In a number of experiments on registration of extensive air showers (EAS) at ultra-high energies, an excessive abundance of muons in comparison with calculations performed on the basis of modern models of hadronic interactions was found, even under the assumption of extremely heavy (iron group nuclei) mass composition of primary cosmic rays (PCR). A recent combined analysis of the data of nine major experiments on detection of EAS muon component performed by the inter-collaboration working group WHISP [1] has shown that the muon excess increases with the increase of primary energy, and this increase is statistically significant.

One of the first experiments where the muon excess was found and a clear indication for its growth with the energy of primary particles was obtained, was the NEVOD-DECOR experiment on investigation of muon bundles in inclined EAS [2, 3]. An approach to the analysis of muon bundle data in this experiment is based on the phenomenology of local muon density spectra (LMDS) at various zenith angles. At large zenith angles, transverse dimensions of the shower in muon component become much larger than typical sizes of the detector which may be considered as a point-like probe. In an individual event with a muon bundle, the local muon density in the point of the device location is estimated. At different zenith angles, substantially different (up to the orders of magnitude) primary energies correspond to the same muon density. Another important point is that the effective area of the event collection is determined not by the detector size but by the cross section of the EAS in the muon component, and reaches square kilometers near the horizon. Simultaneous detection of muon bundles in a wide range of zenith angles gives a possibility to explore a very wide interval of PCR energies (from $\mathrm{PeV}$ to $\mathrm{EeV}$ and higher) in the frame of a single experiment.

In the present paper, results of the NEVOD-DECOR experiment on investigations of muon bundles based on the data accumulated over the period from May 2012 to March 2021 are presented. Statistics of the events more than twice exceeds the data published earlier [2, 3]. A brief description of the experiment and new results of the measurements of the LMDS in the zenith angle interval $40^{\circ}-85^{\circ}$ are shown and compared with simulation results obtained on the bases of the CORSIKA program [4] for two widely used hadronic interaction models: SIBYLL2.3c [5] and QGSJET-II-04 [6] for different assumptions on PCR mass composition.

\section{Experimental data}

Registration of muon bundles in inclined EAS is performed by means of the coordinatetracking detector DECOR [7] which is a part of the Experimental Complex NEVOD [8]. The DECOR was designed for investigations of cosmic rays at large zenith angles. It includes 8 supermodules (SM) deployed in the galleries of the experimental complex building from three sides of the Cherenkov water calorimeter NEVOD. Each SM has an area of $8.4 \mathrm{~m}^{2}$ and consists of eight vertical planes of plastic streamer tube chambers with resistive cathode coating and two-coordinate $(\mathrm{X}, \mathrm{Y})$ external strip readout system. The accuracy of the spatial and angular reconstruction of muon tracks crossing the $\mathrm{SM}$ is better than $1 \mathrm{~cm}$ and $1^{\circ}$, respectively.

Selection of muon bundle events is performed in several stages. At the first (hardware) level the events with at least three (of eight) hit supermodules are saved; the rate of such events is about $0.25 \mathrm{~s}^{-1}$. At a further selection of muon bundles in an off-line regime a fact is used that 
the trajectories of muons formed in the atmosphere at large distances from the setup are practically parallel. First, by means a software program, the events which contain at least 3 quasi-parallel tracks (within $5^{\circ}$-cone) in three different SMs are selected. Then a more hard selection of the events according to zenith and azimuth angles and a certain threshold in the number of found parallel tracks is applied. Final event selection and muon track counting are performed visually by the operators with the help of a specialized program interface. The result of the selection procedure is the list of muon bundles containing event identifiers, estimates of zenith and azimuth angles, program and visual estimates of muon track multiplicities.

In the present work, data accumulated in four long-term series of measurements conducted over the period from 03.05.2012 to 10.03.2021 are used. The total live time of observations amounted to $\sim 58,350 \mathrm{~h}$. In these data, $\sim 99.6$ thousand muon bundles with zenith angles $\theta \geq 55^{\circ}$ and muon multiplicity $m \geq 5$ have been found. The events were selected in two $60^{\circ}$-wide ranges of azimuth angles where most of DECOR SMs (6 of 8) were shielded by the water volume of the Cherenkov calorimeter, readings of only these 6 screened SMs were used for an estimate of the number of muon tracks in a bundle. In such selection conditions, a threshold muon energy is close to $2 \mathrm{GeV}$. Additionally, from a part of the experimental material $(\sim 6,324 \mathrm{~h}) \sim 30.4$ thousand muon bundles with the same minimal muon multiplicity $(m \geq 5)$ but with lower zenith angles $40^{\circ} \leq \theta<55^{\circ}$ have been selected.

Phenomenological dependences of the rate of selected muon bundles on multiplicity and zenith angle are presented in fig. 1 and fig. 2 . As seen from the figures, the available muon bundle data embrace the range of about 5 decades in the event intensity.

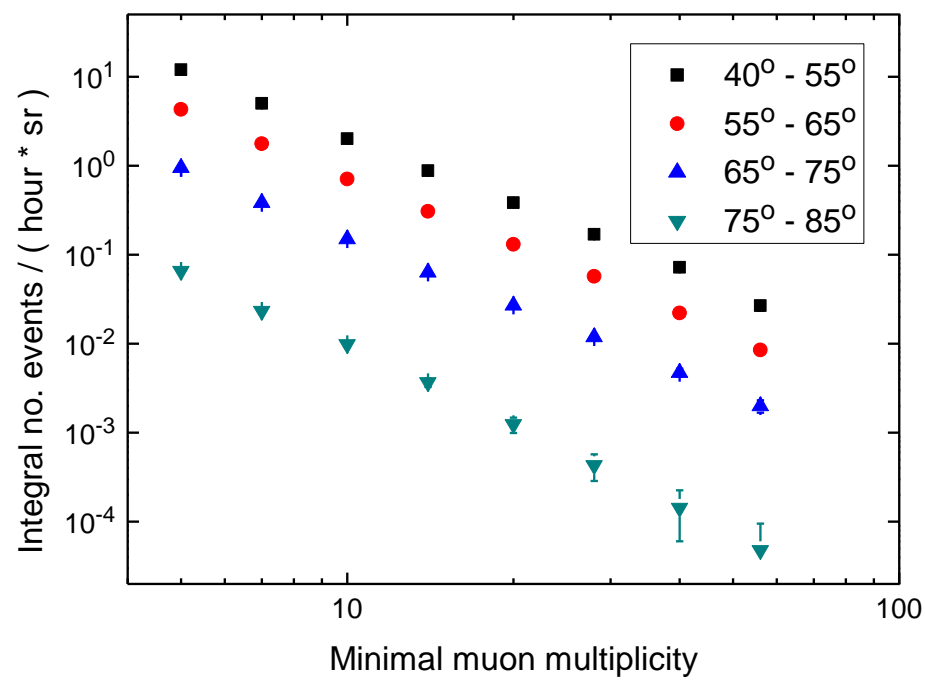

Figure 1: Dependences of the integral intensity of muon bundles on minimal muon multiplicity for several intervals of zenith angle. 


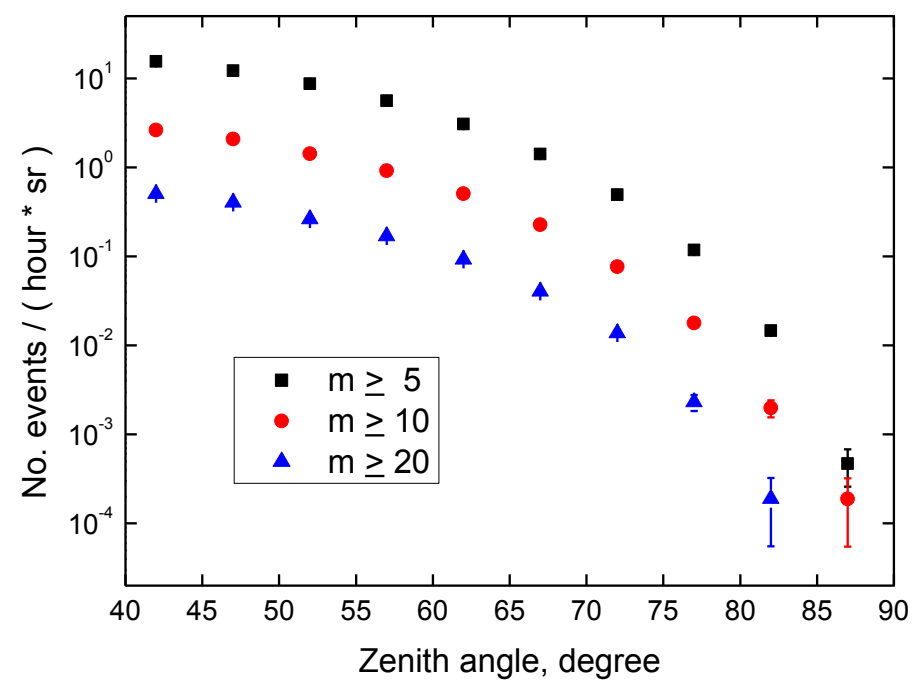

Figure 2: Dependences of the integral intensity of muon bundles on the zenith angle for different minimal muon multiplicities.

\section{Local muon density spectra (LMDS)}

The procedure of reconstruction of local muon density spectra from the experimental data on muon bundles was described in brief earlier [9]. For every muon bundle, an estimate of the mean logarithmic muon density (muon density proxy) in the showers contributing to events with a fixed multiplicity $m$ for a certain arrival direction $(\theta, \varphi)$ was calculated:

$$
D_{\text {est }}=(m-\beta-0.5) / S_{\text {det }}(\theta, \varphi) \text {. }
$$

Here, $\beta \approx 2.1$ is the slope of integral LMDS, $S_{\text {det }}(\theta, \varphi)$ is the total area of six DECOR SMs for this direction. The events were sorted in nine $5^{\circ}$-wide intervals of zenith angles from $40^{\circ}$ to $85^{\circ}$ and several bins of the density estimate $D_{\text {est }}$ (with a constant step in the logarithm of the density), then the matrix of the numbers of the observed events $N_{\text {obs }}(\Delta D, \Delta \theta)$ was formed. The expected matrix of the event numbers was calculated by means of the Monte Carlo technique. Artificial events (muon density, arrival direction) were sampled according to the reference local muon density spectrum model in the form

$$
d F_{0} / d D=C D^{-(\beta+1)} \cos ^{\alpha} \theta
$$

with parameters $\alpha=4.7$ and $\beta=2.1$ tuned to reach a reasonable description of the basic phenomenological distributions in multiplicity and zenith angle. In simulations, Poisson fluctuations of the number of muons that hit supermodules, realistic SM geometry, the efficiency of streamer tube planes and its dependence on the direction, all basic conditions of hardware, software and operator selection have been taken into account. In particular, the probability of a loss of the tracks due to a finite two-track resolution was considered. Then simulated events were treated similar to real ones, and the matrix of the expected numbers of the events $N_{\exp }(\Delta D, \Delta \theta)$ for the above reference LMDS model (2) was constructed. Finally, the experimental estimates of the differential LMDS (multiplied by a smoothing factor $D^{3}$ ) were obtained as 


$$
D^{3} d F / d D=\left[N_{\text {obs }}(\Delta D, \Delta \theta) / N_{\exp }(\Delta D, \Delta \theta)\right] C D^{2-\beta} \cos ^{\alpha} \theta .
$$

The estimates of LMDS for each matrix cell are attributed to certain points $\left(D^{*}, \theta^{*}\right)$, the choice of which has been optimized from a view-point of a robustness of the reconstruction procedure relative to small variations of the parameters $(\alpha, \beta)$ of the reference model. In particular, as $D^{*}$ we use mean logarithmic values of muon densities for corresponding matrix cells obtained from the simulation. The reconstructed local muon density spectra for nine values of zenith angle are represented by the points in fig. 3 .
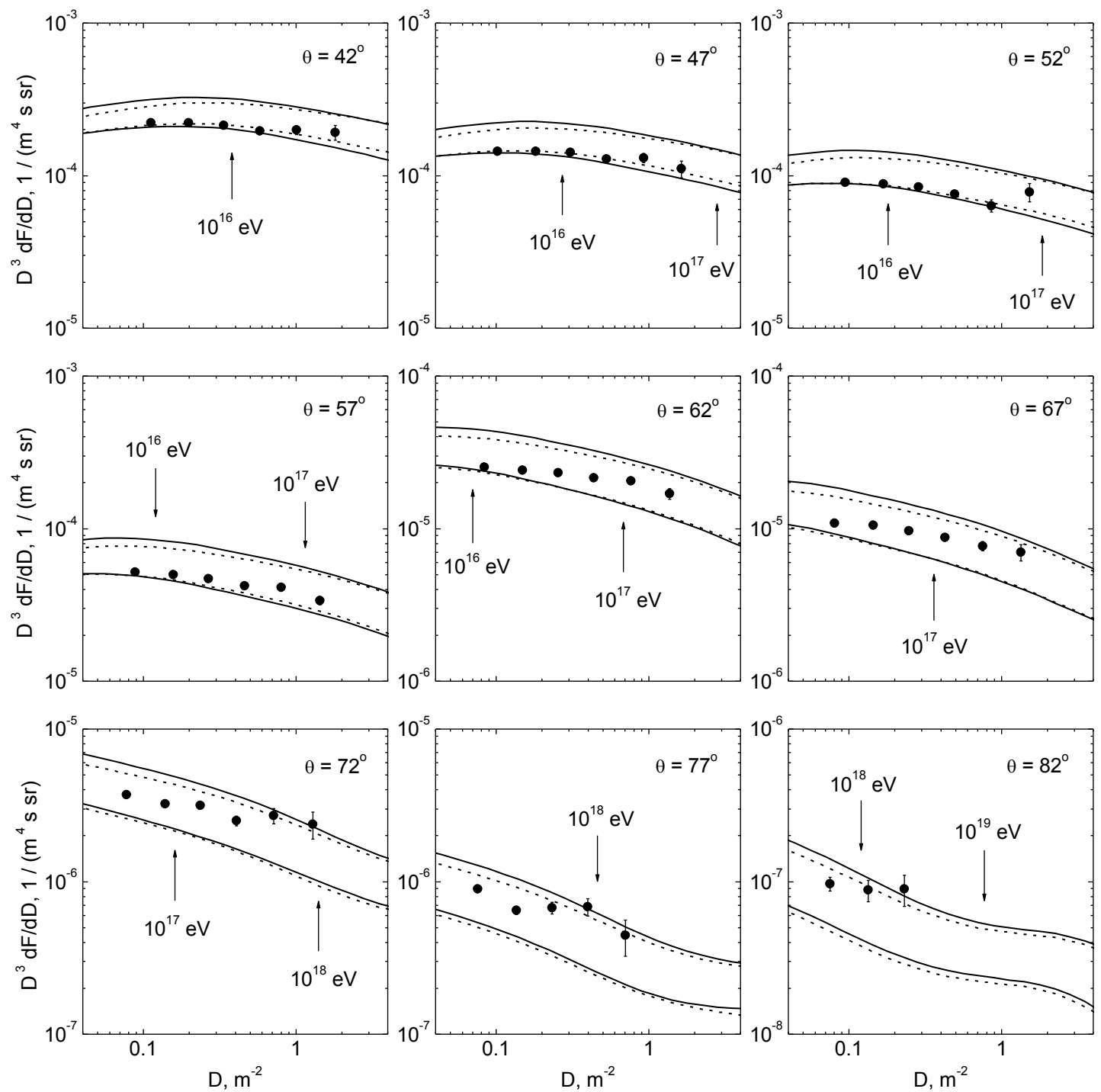

Figure 3: Differential local muon density spectra for nine values of zenith angle (see the labels in the frames). Points: the present experiment. The curves: expected LMDS calculated with the use of the models QGSJET II-4 and SIBYLL 2.3c (solid and dashed curves, respectively) for primary protons (lower pairs of curves in each frame) and iron nuclei (upper pairs of the curves). Arrows indicate typical energies of primary particles (mean logarithmic values estimated for primary protons). 


\section{Expected LMDS calculations}

The local muon density spectrum is formed by the EAS of various energies registered at different (random) distances from the shower axes. In order to calculate the expected integral intensity of the events $F(\geq D)$ in which the local muon density exceeds a preset value $D$, it is necessary to perform the integration over the location of the detector in the shower cross section. For a fixed direction, the integral LMDS may be represented as [2]:

$$
F(\geq D)=\int N(\geq E(\mathbf{r}, D)) d S,
$$

where $\mathbf{r}$ is the point of the detector location in transverse section of the shower, $N(\geq E)$ is the integral spectrum of primary particles, and the minimal energy $E$ of particles contributing to events with muon density greater than $D$ is determined by the solution of the equation:

$$
\rho(E, \mathbf{r})=D \text {. }
$$

Here $\rho(E, \mathbf{r})$ is the lateral distribution function (LDF) of EAS muons (local muon density at the point $\mathbf{r}$ ). From equations (4) and (5), a formula for calculations of the differential LMDS can be readily derived:

$$
d F / d D=\int(d N / d E) d S /[d \rho(E, \mathbf{r}) / d E],
$$

where the relation between $E, D$ and $\mathbf{r}$ is given again by equation (5). More details on the scheme of calculations of the expected LMDS may be found elsewhere [2, 3].

As follows from equations (4) - (6), the expected LMDS is determined by the energy spectrum of PCR and muon LDF, which in its turn depends on the selected hadronic interaction model and atomic weight of the primary nucleus. For calculations of the expected LMDS in this work we used a piece-wise power-law function approximation [3] of the all-particle primary energy spectrum which was based on a recent review of EAS data [10], and two widely used hadronic interaction models: SIBYLL 2.3c [5] and QGSJET II-4 [6]. As two limiting cases of the primary mass composition, protons and iron nuclei were considered. Calculation results for these four combinations for 9 zenith angle values are presented by the curves in fig. 3. As seen from the figure, predictions for LMDS from the two hadronic interaction models are not very different. On the other hand, the difference between the expected LMDS from protons and iron nuclei is significant; it increases with the growth of zenith angle (and, respectively, of primary energy) and reaches a factor $\sim 2.5$ around $10^{17}-10^{18} \mathrm{eV}$.

\section{Comparison and discussion}

Comparison of experimental and calculated LMDS (fig. 3) shows that at moderate zenith angles $\left(\theta=42^{\circ}-57^{\circ}\right)$ data are close to calculations for a light mass composition of PCR. As zenith angle (and, respectively, effective primary energy) increases, a shift of the experimental points toward calculation results for iron is seen, what in principle can be interpreted as a consequence of a heavier mass composition at energies of the order $10^{17} \mathrm{eV}$. At a further transition to the energies about $10^{18} \mathrm{eV}$, data are compatible with calculations only at an assumption of extremely heavy (iron group nuclei) mass composition. Such the assumption contradicts however the results of investigations of the depth of the shower maximum $X_{\max }$ performed by means of the air fluorescence technique [11, 12]. 
For the comparison of the experimental LMDS measured in this work at different zenith angles, it is convenient to use the z-parameter introduced by the WHISP group [1] for the combined analysis of different experiments:

$$
z=\left(\log F^{\mathrm{obs}}-\log F_{\mathrm{p}}^{\mathrm{sim}}\right) /\left(\log F_{\mathrm{Fe}}^{\mathrm{sim}}-\log F_{\mathrm{p}}^{\mathrm{sim}}\right) .
$$

Here $F^{\mathrm{obs}}$ is the result of measurements of some observable proportional to muon abundance (in our case, LMDS), $F_{\mathrm{p}}^{\mathrm{sim}}$ and $F_{\mathrm{Fe}}^{\mathrm{sim}}$ are CORSIKA-based model predictions of this value at assumptions of primary protons and iron nuclei, respectively. In figs. 4 and 5, our results of local muon density spectrum measurements are compared with simulations for two models of hadronic interactions: SIBYLL-2.3c and QGSJET-II-04 in a z-scale. As a measure of the effective primary energy $E_{0}$ (abscissa in the figures), the mean logarithmic energy of primary particles calculated for protons was used; if we would assume primary iron nuclei, the points in the figures would shift to the left by a factor $\sim 1.5$, the ordinate would practically not change. As seen from the figures, the results of LMDS measurements at different zenith angles well agree with each other.

\section{Conclusion}

The DECOR data on local muon density spectra embrace the primary energy range of about three orders of magnitude, and for the both considered hadron interaction models they indicate a fast growth of the z-parameter at primary energies higher than $10^{17} \mathrm{eV}$. Around $10^{18}$ $\mathrm{eV}$ the experimental points are close to the expectation for primary iron nuclei. However, as pointed above, such an assumption contradicts the available data on $X_{\max }$ which favor a light (predominantly proton) mass composition at these energies. Therefore, it seems that the solution of the muon puzzle requires introduction of serious changes in the existing models of hadron interaction at ultra-high energies.

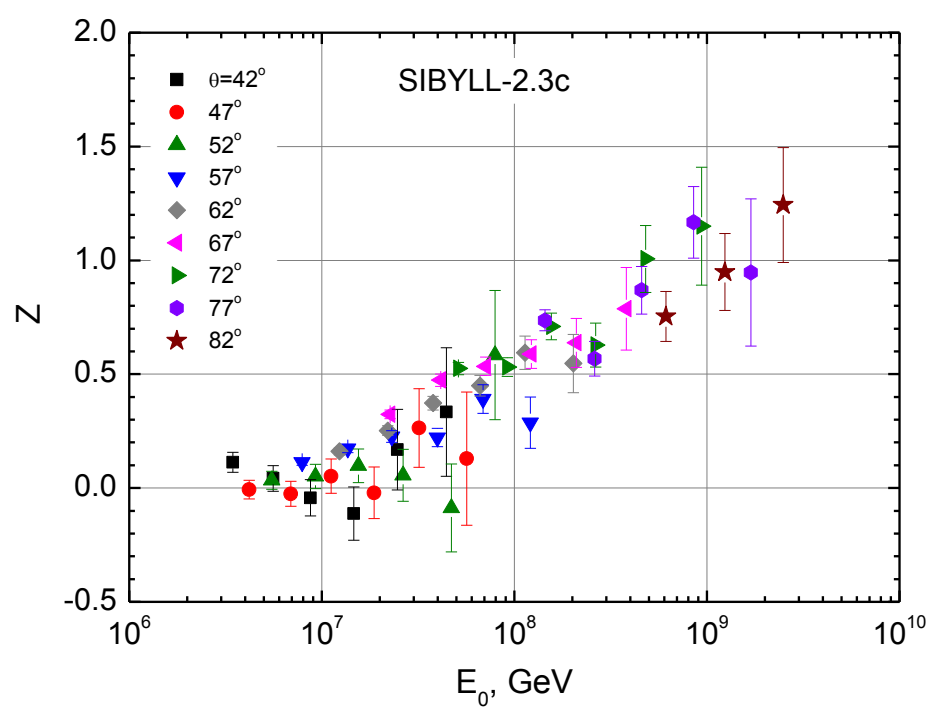

Figure 4: Comparison of the LMDS measured at different zenith angles with the expectation calculated on the basis of SIBYLL-2.3c model. 


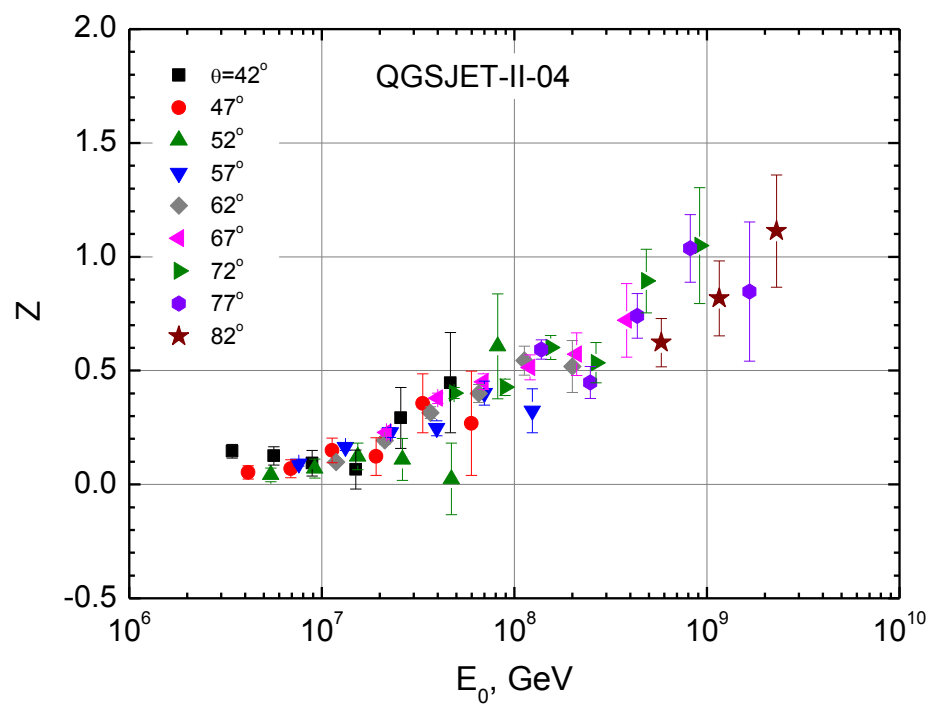

Figure 5: The same as in fig. 4, for QGSJET-II-04 model.

\section{Acknowledgments}

The work was performed at the Unique Scientific Facility "Experimental Complex NEVOD" with the support of the Ministry of Science and Higher Education of the Russian Federation, project "Fundamental problems of cosmic rays and dark matter", No. 0723-20200040. Simulations were performed using the resources of the MEPhI high-performance computing center.

\section{References}

[1] H.P. Dembinski et al., EPJ Web Conf. 210, 02004 (2019), arXiv:1902.08124 [astro-ph.HE].

[2] A.G. Bogdanov et al., Phys. Atom. Nucl. 73, 1852 (2010).

[3] A.G. Bogdanov et al., Astropart. Phys. 98, 13 (2018).

[4] D. Heck et al., Report FZKA 6019, Forschungszentrum Karlruhe GmbH (1998).

[5] F. Riehn et al., Preprint arXiv:1709.07227v1 [hep-ph].

[6] S. Ostapchenko, Phys. Rev. D 89, 074009 (2014).

[7] N.S. Barbashina et al., Instrum. Experim. Techn. 43, 743 (2000).

[8] A.A. Petrukhin, Phys. Uspekhi 58, 486 (2015).

[9] R.P. Kokoulin et al., Phys. Atom. Nucl. 82, 657 (2019).

[10] C. Patrignani et al. (PDG), Review of Particle Physics, Chin. Phys. C 40, 100001 (2016).

[11] J. Abraham et al., Phys. Rev. Lett. 104, 091101 (2010).

[12] R.U. Abbasi et al., Phys. Rev. Lett. 104, 161101 (2010). 